\title{
Du bon usage de l'image dans l'enseignement de la géographie
}

\section{Avertissement}

Les réflexions qui suivent sont nées dans le cadre de séquences de formation initiale d'enseignants et ne prétendent donc pas faire le tour de la question. Il s'agit plutôt de sensibiliser les maîtres débutants aux enjeux de l'usage de l'image dans l'enseignement de la géographie. Le schéma de synthèse qui accompagne ce texte poursuit le même objectif. Par ailleurs le propos de cet article se limite volontairement à l'image visuelle fixe, et plus particulièrement à la photographie. Dans le corps de l'article, nous attribuons au mot «image» un sens restreint: «image» et "photographie» seront pour nous synonymes.

\section{Enjeux et limites de l'usage de l'image}

L'une des ambitions de l'enseignement de la géographie est de donner à voir et à comprendre le monde. Le texte, les statistiques, la carte et la photographie sont les principaux outils dont disposent les élèves et les enseignants pour tenter d'y parvenir. Paradoxalement, alors que l'image semblerait être le média le plus adéquat pour donner à voir le monde, nombre d'enseignants préfèrent réduire celui-ci à l'apparente objectivité des chiffres ou à la linéarité du texte. Même la carte (elle aussi une représentation du monde) est plus volontiers utilisée que l'image par beaucoup d'enseignants, et ce malgré la complexité de ses langages.

Cette retenue face à l'usage de l'image est-elle motivée par l'intuition que toute image est un mensonge, délibéré ou par omission? Les dérives manipulatrices de Timisoara ou de la guerre du Golfe sont dans toutes les mémoires. La déferlante récente des images virtuelles contribue sans doute à désécuriser le spectateur. Mais sans même aller jusqu'à ces exemples extrêmes, quiconque a pris un jour une photographie sait que cette image ne représente qu'un fragment de réalité, dans l'espace et dans le temps; souvent d'ailleurs, il ne retrouve pas dans l'image révélée ce qu'il a voulu ou cru y mettre.

Pourtant, à condition de l'utiliser de manière raisonnée, la photographie est un outil merveilleux qui peut être mis au service d'un enseignement vivant de la géographie. Il faut simplement être conscient du fait que toute image utilisée lors d'une séquence d'enseignement résulte d'une série de choix, lesquels se situent à différents niveaux. L'usage d'une image en géographie ne doit donc pas être innocent, et on ne peut se permettre de l'exploiter n'importe comment.

\section{Production de l'image}

L'auteur d'une photographie cherche à montrer un aspect de la réalité. Il est limité par diverses contraintes, l'une d'elles étant le temps qu'il peut consacrer à attendre la «bonne lumière» ou le «bon moment», qui donneront du sens à son image: une bonne lumière renforce en le soulignant un élément du relief ou au contraire le gomme; le moment où une artère marchande est déserte et ressemble à un décor de théâtre vide d'acteurs peut être le bon moment ou pas selon le but recherché. Quant aux autres contraintes, il peut s'agir de l'accessibilité d'un lieu, des souhaits d'un commanditaire, ou encore des impératifs techniques (par exemple format et sensibilité du film, focale, maîtrise du média). Compte tenu de toutes ces contraintes, l'auteur opère des choix, conscients ou non, qui aboutissent déjà à une certaine interprétation de la réalité. En outre, par sa volonté de témoigner ou de prendre position, l'auteur peut donner un sens au monde tel qu'il le représente.

\section{Utilisation de l'image}

L'utilisateur d'une photographie va lui aussi opérer une série de choix. Celui qui retient telle image plutôt que telle autre choisit, consciemment ou non, de privilégier un certain aspect de la réalité. En mettant ensuite cette image dans un contexte, il lui confère un sens. Il peut d'ailleurs y avoir des distorsions entre le sens que l'auteur a voulu donner à l'image et celui que lui prête l'utilisateur. Une distorsion peut relever d'un manque d'information sur le contexte originel dans lequel la photographie a été faite, ce qui conduit à une ou des erreurs d'interprétation, par exemple lorsqu'un paysage est cadré de telle manière qu'une des caractéristiques essentielles de l'espace n'apparaît pas dans l'image. L'absence de contexte peut même rendre impossible la lecture correcte d'une photographie, par exemple dans le cas d'une photo de structures tectoniques (plis) dépourvue d'échelle. Par ailleurs, et c'est plus grave, une distorsion peut aussi provenir d'un détournement de sens volontaire.

Grégoire Collet et Philippe Hertig, Séminaire pédagogique de l'enseignement secondaire, Avenue de Cour 33, 1007 Lausanne 

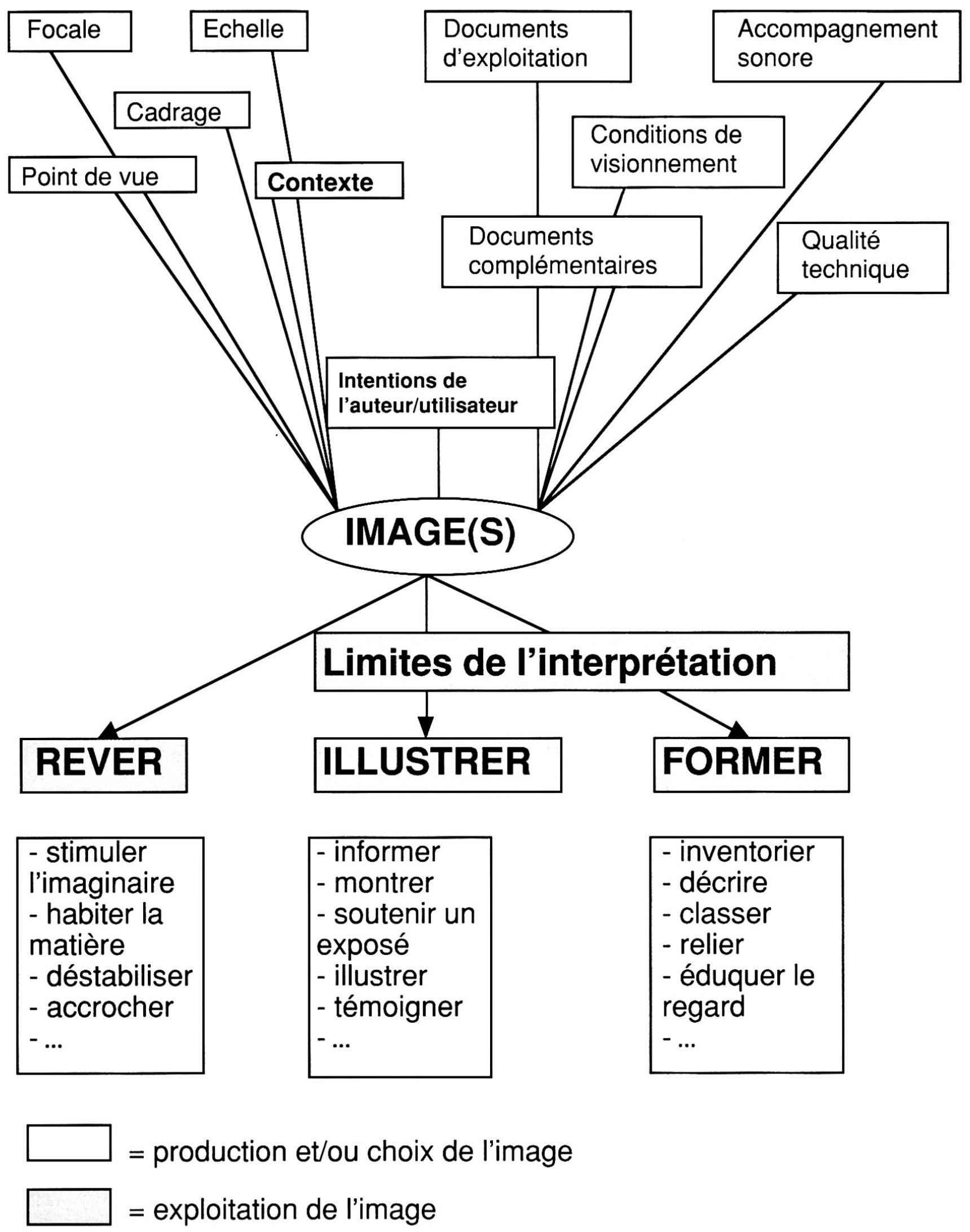

G. Collet/Ph. Hertig. Didactique de la géographie, SPES Lausanne, 1995

Fig.1 Le choix d'une image dans la perspective d'une utilisation pédagogique. 
Tout utilisateur d'une image doit être conscient qu'il ne peut réduire le sens de celle-ci à sa seule interprétation personnelle, puisqu'une image n'est jamais univoque. Il s'agit donc d'être rigoureux dans la dénotation et très prudent avec la connotation. Il faut en outre éviter l'extrapolation; car elle recèle un piège majeur, celui d'être présentée comme une lecture objective de l'image. Par ailleurs, contrairement à beaucoup d'idées préconçues, toutes les précautions évoquées ci-dessus s'appliquent autant à une représentation de paysage qu'à une image au contenu social.

\section{Pratique en classe}

L'enseignant qui envisage d'utiliser l'image en classe devrait avoir à l'esprit les réflexions qui précèdent. C'est plus particulièrement nécessaire lorsqu'il travaille à partir des photographies d'un manuel ou d'un recueil d'images. Mais l'exploitation de ses propres photographies ne le dispense pas de cette réflexion.

Nous distinguons ci-après trois manières complémentaires d'utiliser l'image en classe de géographie: pour former, pour illustrer, pour inviter à rêver. Dans ces trois cas de figure, l'élève est la plupart du temps le récepteur d'une image proposée par l'enseignant.

- Former: cette formation à la lecture de l'image est une éducation du regard, qui devrait faire de l'élève un consommateur critique, conscient du fait qu'une image n'est jamais qu'une représentation partielle et partiale du monde... mais qu'elle est utile, moyennant certaines précautions.

Il s'agit donc de permettre à l'élève d'acquérir une ou des méthode(s) de lecture géographique de l'image; il apprend à distinguer connotation et dénotation, à comparer des images et des points de vue, et, par là, à enrichir et à préciser le contexte. Le travail de dénotation à lui seul implique l'acquisition de plusieurs techniques de lecture de l'image: inventaire des éléments visibles, classement, mise en relation des éléments, division par unités paysagères.

C'est aussi à ce stade que l'élève devrait prendre conscience qu'on ne peut expliquer complètement un paysage sur la base d'une seule photographie: une mise en contexte est indispensable, et il faut avoir recours à d'autres sources d'information (autres photographies, cartes, textes, travail sur le terrain, etc.).

- Illustrer: là réside en apparence l'usage le plus courant et le plus évident de l'image. Il suppose toutefois une grande rigueur intellectuelle de la part de celui qui procède au choix de l'illustration, et il gagne en efficacité si l'èlève-récepteur est formé à la lecture de l'image.

- Inviter à rêver: il s'agit de stimuler et de nourrir l'imaginaire du récepteur. A partir de l'image se construisent des impressions, des ambiances. L'image est le support au travers duquel s'expriment les subjectivités du photographe, de celui qui l'a choisie, et du récepteur, si l'image lui parle. Le récepteur a toute liberté pour créer un contexte à cette image en l'intégrant à ses propres représentations.

Des documents complémentaires ou un accompagnement sonore éventuel peuvent contribuer à esquisser ou à préciser un contexte autour de l'image; des documents d'exploitation (un questionnaire ou une grille d'analyse, par exemple) peuvent guider l'élève dans sa lecture de la photographie.

Dans le cadre scolaire, un autre objectif important est de permettre à l'élève de devenir lui-même un utilisateur averti, par exemple en choisissant des images propres à illustrer de manière significative un exposé. L'acquisition d'un tel savoir-faire peut s'inscrire dans le cours du travail d'éducation du regard évoqué ci-dessus, et elle en représente également un des aboutissements.

\section{En guise de conclusion}

Les idées exposées dans cet article forment l'ossature de la démarche de formation que nous proposons à des enseignants débutants. Cette démarche s'appuie sur de nombreux exemples concrets, mais la place manque ici pour reproduire ces photographies. Nous invitons donc le lecteur à confronter ces quelques réflexions avec sa propre pratique de l'image.

Dans un monde où l'image sert trop souvent de caution à n'importe quel discours, et va même jusqu'à le remplacer, laissant ainsi au spectateur la responsabilité de prendre de la distance, il est du devoir de l'école de donner à l'élève les moyens de décoder l'image et d'en être le moins dupe possible. La géographie, avec ses approches spécifiques, contribue à enrichir le débat et à former les futurs citoyens. A cet égard, nous sommes convaincus de l'utilité et de la richesse de toutes les démarches qui ont recours à l'image et permettent ainsi, au moins en partie, de voir et de comprendre le monde.

\section{Repères bibliographiques}

DIEUDONNE, D., CRAMPON, J.-P., LABRUNE, G. (1991): Histoire, Géographie, Méthodes et techniques, Nathan, Paris.

FERRAS, R., CLARY, M., DUFAU, G. (1993): Faire de la géographie, Belin, Paris.

GRUJARD, M., LE ROUX, A., NOVE, A. (1988): Regarder et comprendre un paysage, Calmann-Lévy.

MERENNE-SCHOUMAKER, B. (1987): Lire les paysages, La Documentation photographique, Multiple no 88, La Documentation française, Paris. 